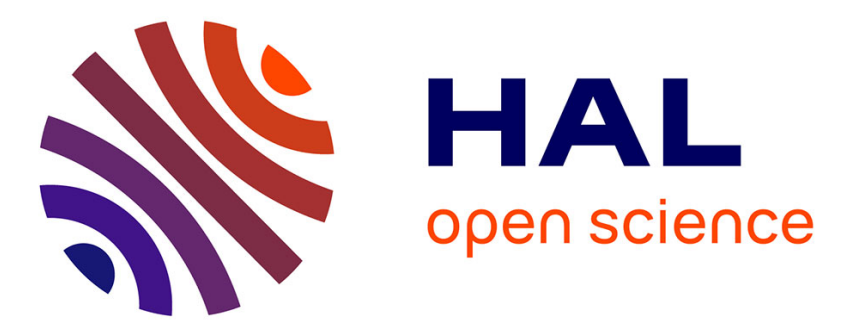

\title{
Conditions d'utilisation, sous hautes pressions isostatiques, de la méthode d'inductance pour la mesure de la compressibilité des solides
}

\author{
A. Lacam, J. Peyronneau, D. Vo Thanh
}

\section{- To cite this version:}

A. Lacam, J. Peyronneau, D. Vo Thanh. Conditions d'utilisation, sous hautes pressions isostatiques, de la méthode d'inductance pour la mesure de la compressibilité des solides. Revue de Physique Appliquée, 1975, 10 (1), pp.35-42. 10.1051/rphysap:0197500100103500 . jpa-00243876

\section{HAL Id: jpa-00243876 https://hal.science/jpa-00243876}

Submitted on 1 Jan 1975

HAL is a multi-disciplinary open access archive for the deposit and dissemination of scientific research documents, whether they are published or not. The documents may come from teaching and research institutions in France or abroad, or from public or private research centers.
L'archive ouverte pluridisciplinaire HAL, est destinée au dépôt et à la diffusion de documents scientifiques de niveau recherche, publiés ou non, émanant des établissements d'enseignement et de recherche français ou étrangers, des laboratoires publics ou privés. 


\title{
CONDITIONS D'UTILISATION, SOUS HAUTES PRESSIONS ISOSTATIQUES, DE LA MÉTHODE D'INDUCTANCE POUR LA MESURE DE LA COMPRESSIBILITÉ DES SOLIDES
}

\author{
A. LACAM, J. PEYRONNEAU et D. VO THANH \\ Centre National de la Recherche Scientifique 1, place Aristide-Briand, 92190 Bellevue, France
}

(Reçu le 24 avril 1974, révisé le 30 mai 1974, révisé le 13 septembre 1974)

\begin{abstract}
Résumé. - La compressibilité des solides, soumis à des hautes pressions isostatiques, est mesurable directement à partir des variations d'inductance d'une bobine solidaire de la surface de l'échantillon. Toutefois, certaines conditions expérimentales sont requises. Ces dernières sont analysées en détail. L'une des plus importantes, notamment en milieu compresseur liquide, concerne l'adhérence sur le support. Par ailleurs, des interactions électromagnétiques, entre la bobine et l'enceinte haute pression, peuvent perturber les mesures. Il est démontré théoriquement et vérifié expérimentalement que ces interactions peuvent être rendues pratiquement indécelables. L'emploi d'un compresseur liquide et la mesure in situ de la pression contribuent largement à la sensibilité de la méthode et assurent une bonne reproductibilité des mesures. Application de la méthode au verre Pyrex.
\end{abstract}

\begin{abstract}
The compressibility of solids, subjected to hydrostatic pressures, is determined by the measurement of the inductance of a coil in intimate contact with the surface of the sample. However, it is necessary to observe certain experimental conditions, which are closely examined. Good adherence between the coil and its support is of major importance, especially in a liquid pressure transmitting medium. Measurements are also upset by electromagnetic interactions between the coil and the pressure vessel. It was found theoretically and verified experimentally that these interactions can be cancelled. Two experimental factors : the use of a liquid transmitting medium and the measurement in situ of the presure, are of major importance for the reproductibility and the sensibility of the method. Application to the Pyrex glass.
\end{abstract}

1. Introduction. - Parmi les études expérimentales, effectuées sur les propriétés physiques des solides sous haute pression, la mesure précise de la variation de volume du solide comprimé demeure l'un des problèmes technologiques des plus difficiles à résoudre.

Les premières tentatives dans ce domaine ont été effectuées, semble-t-il, vers la fin du siècle dernier. Depuis, diverses méthodes ont été utilisées, indirectes pour la plupart et qui souvent impliquaient la prise en considération de termes correctifs mal connus limitant la précision. C'est le cas par exemple des méthodes: ultrasonore $[1,2]$, par jauges de contrainte [3, 4], piézométrique de Bridgman $[5,6,7]$ et de déplacement du piston $[8,9,10,11]$. L'utilisation des rayons $\mathrm{X}$ permet d'observer directement la variation, sous pression, des paramètres du réseau cristallin et d'avoir ainsi accès à la variation du volume. Malheureusement, ce type d'observation oblige, dans la plupart des cas, à travailler en milieu compresseur solide $[12,13,14,15]$. C'est alors la pression qui est mal définie par suite des frottements et des gradients internes. Les observations directes, au moyen d'un dispositif optique, n'ont plus qu'une valeur historique compte tenu de leur limitation en pression [16]. Il en est de même de la méthode employée par Bridgman [17] pour mesurer la compressibilité de l'acier, car elle n'est pas transposable à d'autres matériaux. Plus récemment, Lincoln et Ruoff [18] ont imaginé une nouvelle technique directe, qui paraît très précise, mais qui se trouve limitée en pression. Sa mise en œuvre semble, par ailleurs, des plus délicates.

La présente étude a pour but principal de dégager les conditions expérimentales qui permettent de mesurer avec exactitude et reproductibilité la compressibilité des solides soumis à des pressions parfaitement isostatiques. Cette dernière condition implique l'utilisation d'un milieu compresseur fluide.

La méthode utilisée fait appel aux variations d'inductance d'un bobinage, intimement lié à la surface de l'échantillon cylindrique, lorsque ce dernier se déforme sous l'action de la pression. Dans ces conditions, la relation qui relie l'inductance aux configurations géométriques du bobinage est la suivante :

$$
L=10^{-3} \cdot \pi^{2} \cdot K\left(\frac{d_{\mathrm{e}}}{l_{\mathrm{b}}}\right) N^{2} \cdot d_{\mathrm{e}}-L_{\text {cor }}
$$


dans laquelle :

$d_{\mathrm{e}}=$ diamètre de l'échantillon,

$l_{\mathrm{b}}=$ longueur utile du bobinage,

$K$ est un paramètre qui dépend du rapport $d_{\mathrm{e}} / l_{\mathrm{b}}$,

$L_{\text {cor }}$ représente des termes correctifs accessibles au calcul. Ils sont, par ailleurs, indépendants de la pression,

$N=$ nombre total de spires du bobinage.

Il faut remarquer que si le solide est isotrope et si de plus il est comprimé par un milieu isostatique, il se déformera homothétiquement. Il en résulte alors que $d_{\mathrm{e}} / l_{\mathrm{b}}$ et $K$ sont indépendants de la pression. De plus, si le matériau testé est isolant et non magnétique, l'effet de peau est négligeable.

Dans ces conditions on peut écrire que :

$$
L=\text { Cte. } d_{\mathrm{e}} \quad L \propto V^{1 / 3} .
$$

On dispose donc d'une méthode directe pour la mesure de la compressibilité des solides lorsque les conditions énoncées ci-dessus sont remplies.

Giardini, Poindexter et Samara [20] furent parmi les premiers à mettre en œuvre cette méthode. Utilisant un dispositif de compression polyédrique, fonctionnant en milieu compresseur solide, ils ont déterminé la compressibilité de solides et mesuré des variations de volume dues à des transitions polymorphiques induites par la pression. Il ne semble pas que la précision de leurs mesures soient parmi les meilleures, notamment aux basses pressions. En effet, une bonne précision ne peut être espérée que si :

a) l'échantillon se déforme homothétiquement. Ce qui est rarement obtenu avec un milieu compresseur solide,

b) les variations de géométrie du bobinage et de l'échantillon sont identiques,

c) les interactions électriques ou magnétiques entre le bobinage et l'enceinte haute pression sont négligeables.

Alors que la condition $a$ ) est facile à réaliser par l'emploi d'un fluide comme transmetteur, les points $b$ ) et $c$ ) furent les plus difficiles à solutionner. La suite de cette étude montrera les diverses solutions qui ont été apportées à ces problèmes techniques.

2. Dispositifs expérimentaux. -2.1 ApPareIllage HAUTE PRESSION. - L'appareil utilisé est du type classique piston/cylindre. Le milieu compresseur était constitué par un mélange équivolumétrique de n-pentane et d'iso-pentane qui, d'après Barnett et Bosco, transmet isostatiquement les efforts jusqu'à des pressions de l'ordre de $60 \mathrm{kbar}$ [21].

L'enceinte haute pression est constituée par une chambre multifrettes en acier. Elle peut supporter des pressions allant jusqu'à 28 kbar. Son alésage est voisin de $20 \mathrm{~mm}$ et la course utile du piston de l'ordre de $10 \mathrm{~cm}$. La chambre de compression est fermée à sa partie supérieure par un obturateur fixe comportant les passages de courant.
Les variations d'inductance à mesurer sont relativement peu conséquentes d'où l'importance primordiale du positionnement relatif des divers conducteurs électriques. La position de ces derniers doit rester fixe pendant toute la durée de l'expérience sous peine d'introduire des variations parasites. Pour maintenir cette géométrie globale constante nous avons utilisé, pour pénétrer dans le milieu sous pression, des conducteurs thermocoaxes. Il s'agissait de bifilaires en cuivre ayant une gaine en acier inoxydable. L'isolement est assuré par de la magnésie compactée. Il s'est avéré à l'expérience que ces éléments assurent une très bonne étanchéité au fluide compresseur. Ils sont, par ailleurs, quasi non-inductifs par construction.

2.2 Mesure DE LA PRESSION. - Elle est effectuée directement dans le fluide compresseur au moyen de jauges résistantes en fil de manganine. La valeur initiale des résistances est voisine de $120 \Omega$. Le fil de manganine, isolé par un double guépage de soie, est bobiné non inductif sur un support souple de papier pour éviter les tensions mécaniques.

Les jauges ont été préalablement stabilisées par des traitements thermiques comprenant des maintiens de longue durée à $140^{\circ} \mathrm{C}$ alternés avec des trempes à l'azote liquide. Une mise sous pression à $25 \mathrm{kbar}$ terminait le processus de stabilisation. Les jauges ainsi stabilisées, puis étalonnées au moyen d'une balance de pression, peuvent être considérées comme des étalons secondaires. Elles permettent alors d'effectuer des mesures avec une très grande définition et une bonne reproductibilité $[22,23,24]$.

La figure 1 montre les variations du coefficient de pression $\Delta R / R_{0} . P$ en fonction de ce paramètre.

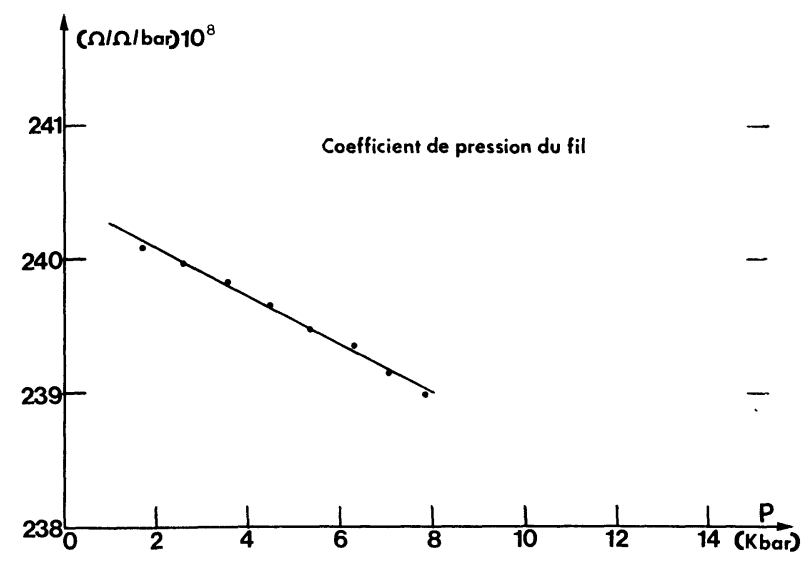

FIG. 1. - Variations du coefficient de pression $\Delta R / R_{0} . P$ des jauges en fonction de $P$.

La figure 2 traduit les corrections à effectuer pour tenir compte de ces variations.

D'une manière générale, deux jauges étaient placées dans l'enceinte ; ceci afin de diminuer les risques d'erreurs dues à un fonctionnement imparfait de 
l'électronique de mesure ou aux jauges elles-mêmes. Cette disposition rendait possible une intercomparaison permanente des mesures.

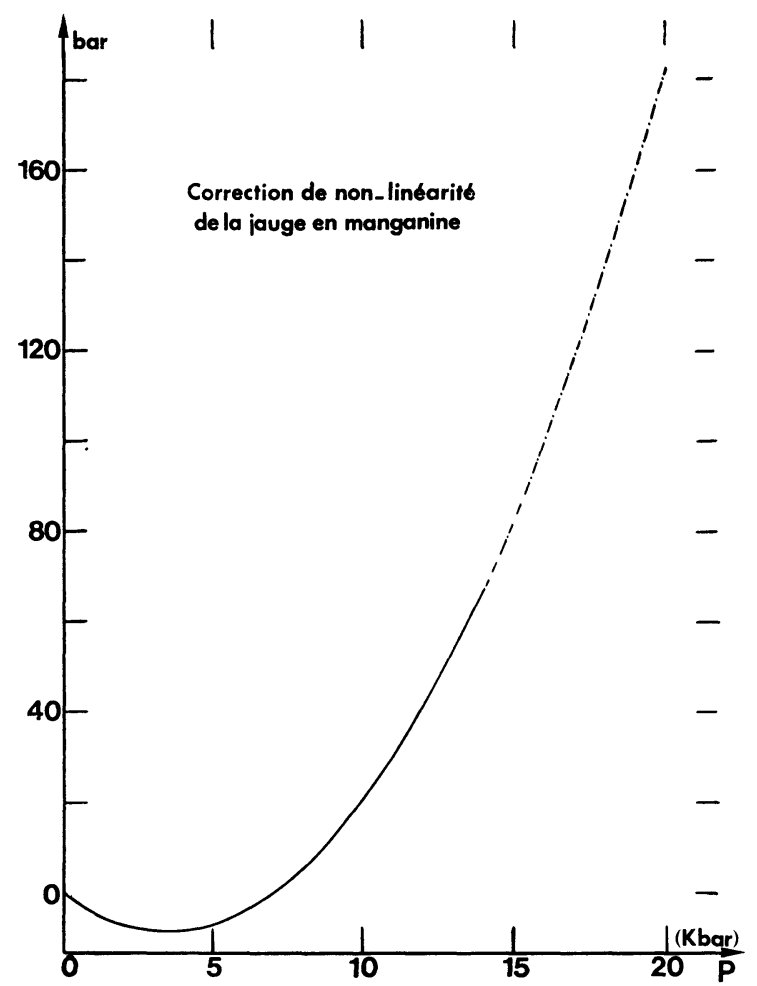

Fig. 2. - Courbe de correction de non-linéarité des jauges.

Les pressions sont déterminées de la manière suivante : l'une des jauges est reliée directement à un ohmètre numérique ayant une résolution de $0,002 \Omega$. Cet appareil permet de suivre visuellement l'évolution de l'expérience. La seconde jauge est insérée dans un montage en pont de résolution $0,0005 \Omega$. Les mesures précises sont faites avec ce dispositif.

2.3 Mesure DE L'INDUCTANCE. - L'appareil de mesure est un pont d'inductance General Radio Company, type 1632 A.

La définition maximale de ce pont est de $10^{-4} \mu \mathrm{H}$ pour un bobinage ayant une résistance inférieure à $5 \Omega$ et une inductance n'excédant pas $100 \mu \mathrm{H}$. La décade suivante, dix fois moins sensible, tolère une résistance de $50 \Omega$. Par ailleurs, la sensibilité du pont augmente avec la fréquence de mesure et avec le coefficient de surtension $L / R$.

3. Le bobinage inductif. - Le problème technique le plus important, et qui s'est révélé le plus difficile à résoudre, a été celui d'obliger le bobinage à suivre exactement les déformations de l'échantillon. Si cette condition n'est pas respectée il s'en suit automatiquement une mauvaise reproductibilité des résultats, quand toutefois ceux-ci ne sont pas complètement erronés. Pour solutionner ce problème, diverses techniques de fabrication ont été éprouvées, à savoir :

3.1 Bobinages Classiques a fils fins. - Ce type de bobinage ne pose aucun problème de fabrication. Par ailleurs, il permet d'obtenir un coefficient de surtension $L / R$ relativement élevé. En revanche, il n'existe aucune liaison entre lui et l'échantillon. Il est donc nécessaire de recouvrir l'ensemble par un écran étanche au fluide compresseur qui sert alors de transmetteur intermédiaire. Ce type de bobinage ne s'est pas révélé satisfaisant, dans nos conditions d'expérience, et fait perdre une partie des avantages que procure l'utilisation d'un transmetteur liquide. Toutefois, Giardini et ses Collaborateurs [20, 25] mentionnent l'emploi d'un tel type de bobinage en milieu compresseur solide. D'après ces auteurs, la précision varierait de 5 à $15 \%$ en fonction des conditions de pression; aucune mesure cohérente n'étant possible au-dessous de $5 \mathrm{kbar}$.

3.2 BOBINAGES OBTENUS PAR ÉVAPORATION SOUS VIDE. - Le bobinage est obtenu en évaporant, à travers un cache métallique, une couche d'un métal bon conducteur électrique. De telles couches, qui présentent l'avantage d'épouser parfaitement la surface de l'échantillon, ont obligatoirement, sous peine d'hétérogénéités dans la masse, une épaisseur limitée. Il en résulte une résistance électrique globale importante. Toutefois, leur inconvénient majeur est d'avoir une adhérence relativement faible sur la surface de l'échantillon. La liaison peut être renforcée, comme dans le cas précédent, en recouvrant l'ensemble d'une couche étanche protectrice. Quelques résultats positifs ont été obtenus par cette méthode. Toutefois le taux de réussite est assez faible.

\subsection{BOBINAGES OBTENUS PAR RÉDUCTION CHIMIQUE} A CHAUD. - En vue d'obtenir une meilleure adhérence, nous avons été conduits à tester des bobinages obtenus par réduction chimique à chaud d'une peinture métallique à base de métaux précieux. Lors de la réduction, il se produit une légère diffusion, quelques $\AA$, du métal dans le support. Cette dernière est néanmoins suffisante pour assurer une adhérence très importante. Expérimentalement, de tels bobinages ont été utilisés jusqu'à des pressions de l'ordre de $20 \mathrm{kbar}$ sans que nous n'ayons noté de phénomènes parasites, tels que coupures ou décollement des spires.

Ce procédé nous a permis d'obtenir des couches homogènes ayant une épaisseur de l'ordre de $30 \mu$. C'est-à-dire des épaisseurs environ cinq fois plus importantes que lors des évaporations sous vide. Il en résulte un coefficient de surtension acceptable et une résistance électrique peu élevée. C'est le procédé qui nous a fourni les résultats les plus satisfaisants. Néanmoins, il n'est utilisable qu'avec des échantillons silicatés ou des oxydes. En effet, les fondants contenus dans la peinture, ne réagissent qu'avec ces types de matériaux. 
Le tableau I résume les caractéristiques des trois types de bobinages :

\section{TABLEAU I}

\begin{tabular}{|c|c|c|c|}
\hline $\begin{array}{c}\text { Type } \\
\text { de bobinage }\end{array}$ & Fils fins & Evaporation & Réduction \\
\hline $\begin{array}{l}L / R \\
\text { Adhérence } \\
\text { Nature de } \\
\text { l'échantillon }\end{array}$ & $\begin{array}{l}\text { bon } \\
\text { mauvaise } \\
\text { tous }\end{array}$ & $\begin{array}{l}\text { médiocre } \\
\text { moyenne } \\
\text { tous }\end{array}$ & $\begin{array}{l}\text { assez bon } \\
\text { bonne } \\
\text { silicates oxy- } \\
\text { des }\end{array}$ \\
\hline
\end{tabular}

4. Interactions avec l'enceinte haute pression. - Des expériences préliminaires, effectuées jusqu'à des pressions de $20 \mathrm{kbar}$, avec des échantillons de diverses natures nous ont montré que les mesures peuvent être perturbées par l'environnement. En effet, le pont d'inductance fonctionne en courant alternatif ; de ce fait, la bobine se trouve soumise à un champ magnétique variable provoquant des interactions avec l'enceinte haute pression en acier. Les interactions sont dues à deux phénomènes indépendants : l'une est d'origine électrique et l'autre magnétique. Le problème consiste donc à voir s'il est possible soit de les calculer avec précision, soit de les diminuer de telle manière qu'elles deviennent négligeables ou nulles.

4.1 ETUde THÉORIQUE. - Divers cas sont à examiner selon la nature de l'échantillon :

4.1 .1 Ecran métallique : interaction électrique.Si l'on place une bobine à l'intérieur d'un cylindre conducteur non magnétique, ce dernier est parcouru par les courants induits de Foucault. Ensuite, une action mutuelle apparaît entre ce courant et celui de la bobine. Tout se passe comme dans un transformateur. A partir des équations classiques des transformateurs nous déduisons :

$$
L=L_{0}-\frac{M^{2} l_{2} \omega^{2}}{\left(r_{2}^{2}+\omega^{2} l_{2}^{2}\right)}=L_{0}-L_{\mathrm{i} . \mathrm{e}}
$$

expression dans laquelle $L$ et $L_{0}$ sont les inductances en présence et en l'absence de l'écran. $r_{2}$ et $l_{2}$ la résistance et l'inductance de l'écran. $M$ est l'inductance mutuelle entre bobine et écran.

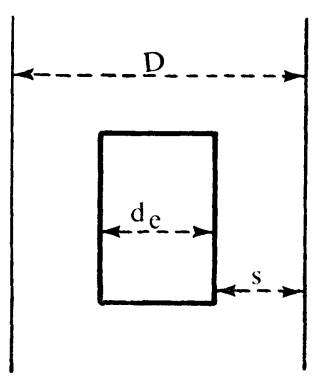

4.1.2 Ecran magnétique : interaction magnétique. $\mathrm{Si}$, maintenant, l'écran est un isolant magnétique, les courants de Foucault n'existent plus. En revanche, l'écran se trouve aimanté par le champ magnétique de la bobine. Cette aimantation n'est plus négligeable comme c'était le cas avec un écran non magnétique. Ce fait est dû à la grande valeur de la perméabilité magnétique de ces corps. Elle contribue à augmenter le flux d'induction à travers la section $S$ du solénoïde :

$$
L=L_{0}+\frac{B_{\mathrm{i} \cdot \mathrm{m}} \cdot S}{I}=L_{0}+L_{\mathrm{i} \cdot \mathrm{m}}
$$

dans laquelle $B_{\mathrm{i} . \mathrm{m}}$ est l'induction magnétique à l'intérieur du solénoïde produite par l'écran aimanté. $I$ est l'intensité du courant dans le solénoïde.

4.1.3 Ecran conducteur magnétique. - Dans ce cas, les deux interactions précédentes se superposent. Comme elles sont relativement faibles, il est possible d'écrire, en première approximation, que :

$$
L=L_{0}+\frac{B_{\mathrm{i}, \mathrm{m}} \cdot S}{I}-\frac{M^{2} l_{2} \omega^{2}}{r_{2}^{2}+\omega^{2} l_{2}^{2}}=L_{0}+L_{\mathrm{i}, \mathrm{m}}-L_{\mathrm{i} . \mathrm{e}} .
$$

4.1.4 Conséquences. - Des considérations théoriques, nous pouvons tirer les conséquences suivantes :

\section{TABleAU II}

\section{Type d'écran \\ Conducteur non magnétique (1)}

Sens de la variation

$$
L / L_{0}<1
$$

\section{Dépendance de $\omega$}

$$
\omega_{1}>\omega_{2} \rightarrow L_{\omega_{2}}>L_{\omega_{1}}
$$

Indépendant

Influence des proprié- $\rho_{1}>\rho_{2} \rightarrow L_{01}>L_{02}(* * *)$ tés de l'écran

Influence de la dis- $L_{\mathrm{i} . \mathrm{e}} \propto M^{2} \propto 1 / S^{4}\left(^{*}\right)$ tance

(*) D'après Grover [26].

$(* *)$ D'après la loi de Biot et Savart.

$(* * *) \rho$ est la résistivité électrique de l'écran.

$(* * * *) \mu$ est la perméabilité magnétique de l'écran.
Conducteur magnétique (3)
$L / L_{0}<L / L_{0}<L / L_{0}$

(1) (2)

$\omega_{1}>\omega_{2} \rightarrow L_{\omega_{2}}>L_{\omega_{1}}$

Conformément aux actions conjuguées
$L_{\text {i.e }} \propto 1 / S^{4}$

$L_{\mathrm{i}, \mathrm{m}}$ 
5. Résultats expérimentaux. - La vérification expérimentale des données théoriques a été effectuée en utilisant des écrans de natures suivantes :

- Le cuivre et le laiton pour les écrans conducteurs non magnétiques. tique.

- Le ferrite de manganèse pour l'isolant-magné-

- L'acier pour le cas général.

La figure 3 illustre les résultats obtenus avec ces différents matériaux. Il est possible d'en tirer les conclusions suivantes :

a) $\left(L / L_{0}\right)_{\text {lai ton }}$ et $\left(L / L_{0}\right)_{\text {cuivre }}<1$,

$$
\begin{aligned}
& \left(L / L_{0}\right)_{\text {ferrite }}>1, \\
& \left(L / L_{0}\right)_{\text {laiton }}<\left(L / L_{0}\right)_{\text {acier }}<\left(L / L_{0}\right)_{\text {ferrite }} .
\end{aligned}
$$

De plus $\left(L / L_{0}\right)_{\text {acier }}>1$. Ce qui entraîne pour l'acier $L_{\mathrm{i}, \mathrm{m}}>L_{\mathrm{i} . \mathrm{e}}$.

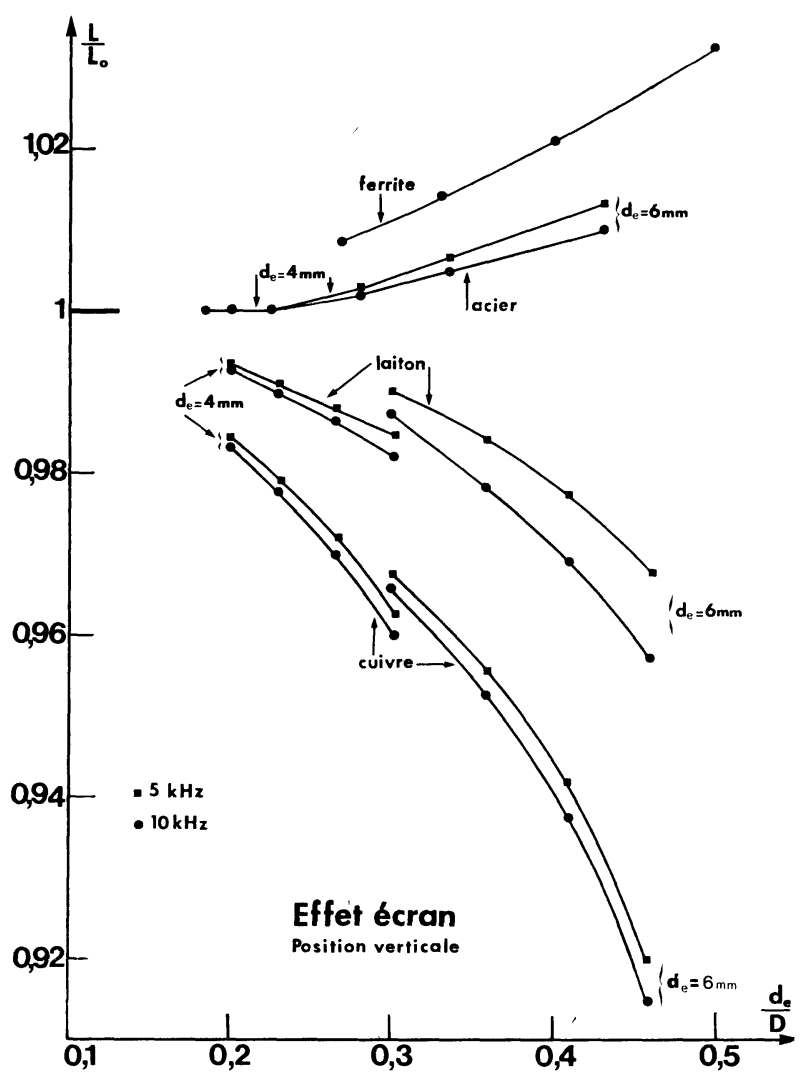

Fig. 3. - Effet d'écran pour divers matériaux et différentes conditions géométriques.

b) Dans le laiton, le cuivre et l'acier :

$$
L_{5 \mathrm{kHz}}>L_{10 \mathrm{kHz}} \text {. }
$$

Les mesures sont indépendantes de la fréquence avec un écran de ferrite.

c) $L_{\text {lai ton }}>L_{\text {cuivre }}$ puisque $\rho_{\text {lai ton }}>\rho_{\text {cuivre }}$.

d) Les deux interactions décroissent avec le rapport $d_{\mathrm{e}} / D$. ( $D$ étant le diamètre intérieur de l'écran.)

L'étude théorique se trouve donc entièrement confirmée par les résultats expérimentaux, du moins en ce qui concerne les expériences effectuées à la pression atmosphérique.

5.1 Choix D'Une solution. - L'enceinte haute pression, dans le cas de nos conditions d'expérience, joue le rôle d'écran. Comme elle est en acier, il est prévisible que les deux interactions envisagées vont se conjuguer. Il apparaît, par ailleurs, difficile de les évaluer à partir de la configuration géométrique des éléments en présence compte tenu des modifications qu'entraîne l'application de la pression. Faute de pouvoir évaluer les interactions, la seule solution accessible est de les rendre négligeables ou nulles.

La figure 3 et le tableau II montrent clairement que le seul moyen d'action réside dans la possibilité de réduire le diamètre de l'échantillon, ceci pour une enceinte d'alésage donné. D'après la figure 3 , nous voyons qu'un écran en acier ne perturbe plus les mesures lorsque les diamètres de la bobine et de l'écran sont dans un rapport inférieur à 0,225 . Dans ces conditions, les interactions ne deviennent plus décelables expérimentalement. L'alésage de la chambre haute pression étant de $20 \mathrm{~mm}$, nous avons donc été conduits à adopter un diamètre d'échantillon n'excédant pas $4,5 \mathrm{~mm}$. Une étude semblable sur les effets d'extrémités du solénoïde nous a montré que, pour les dimensions précitées, une distance minimum de $15 \mathrm{~mm}$ était indispensable pour éliminer les interactions vis-à-vis des pièces métalliques avoisinantes. C'est cette géométrie globale que nous avons adoptée pour tester la méthode sous haute pression.

Des solénoïdes répondant aux conditions énumérées ci-dessus nous ont permis d'obtenir, pour un pas fixe $\mathrm{du}$ bobinage, un coefficient de surtension de l'ordre de 0,5 . Selon la longueur du bobinage, compatible soit avec la précision recherchée soit avec les conditions géométriques, l'inductance pouvait être comprise entre 1,5 et $5,5 \mu \mathrm{H}$, les résistances ohmiques correspondantes s'échelonnaient alors entre 3 et 11 ohms. Pratiquement, la majeure partie des échantillons expérimentaux utilisés avaient une inductance de 3,5 $\mu \mathrm{H}$ et une résistance de $7 \mathrm{ohms}$. Dans nos conditions d'expérience, la résistance ohmique des connexions avoisine $0,5 \Omega$ et leur inductance $0,8 \mu \mathrm{H}$. Cette dernière s'est avérée parfaitement invariable en cours d'expérience.

Les fréquences de mesure ont été uniquement de 5 et $10 \mathrm{kHz}$. Pour des valeurs plus faibles, la sensibilité du pont de mesure diminue considérablement par suite de la faible valeur du coefficient de surtension.

5.2 Application de la méthode au Pyrex. - Pour des raisons pratiques et économiques nous avons effectué les études préliminaires sur le verre Pyrex. Celui-ci, de plus, a une compressibilité relativement conséquente et les peintures conductrices y adhèrent bien.

Les échantillons étaient obtenus en tronçonnant, perpendiculairement à l'axe d'étirage, des baguettes 
d'environ 1,20 m de longueur. Tous les échantillons proviennent d'un même lot de baguettes.

Les mesures de reproductibilité ont été effectuées sur des échantillons de $4 \mathrm{~mm}$ de diamètre, valeur qui correspond à des interactions électromagnétiques négligeables. Conformément aux études théoriques des interactions, les résultats sur des échantillons de 15,25 et $40 \mathrm{~mm}$ de longueur se sont révélés identiques. Nous avons donc adopté, en règle générale, une longueur de $25 \mathrm{~mm}$ qui nous assurait le maximum de sécurité, en ce qui concerne les effets d'extrémité, tout en conservant une valeur acceptable de l'inductance globale du bobinage.

Les bobinages ont été obtenus par réduction à chaud de peintures conductrices à base d'argent. Pour des bobinages de $25 \mathrm{~mm}$ la résistance ohmique était voisine de $7 \Omega$ et l'inductance de $3,5 \mu \mathrm{F}$.

Toutes les mesures ont été faites aux fréquences de 5 et $10 \mathrm{kHz}$. Dans nos conditions d'expérience, pour des pressions maintenues fixes, l'erreur relative de mesure sur l'inductance est de l'ordre de $5 \times 10^{-4}$. Les déterminations étaient faites en procédant par palliers successifs de pressions espacés d'environ 1 kbar. Les pressions les plus élevées se situaient vers $20 \mathrm{kbar}$.

Il est à noter que les courbes à la décompression n'ont pratiquement jamais été accessibles dans leur totalité. Ce fait semble dû à des contraintes prenant naissance au niveau de la jonction entre le bobinage et l'échantillon auxquelles s'ajoute un écrouissage du métal sous pression. Il en résulte une détérioration de la pellicule métallique qui se rompt lorsque la décompression atteint une certaine fraction de la valeur de la pression maximale atteinte. Néanmoins, un certain nombre de points sont toujours accessibles en début de décompression, généralement sur une plage de l'ordre de $5 \mathrm{kbar}$, voire davantage dans certains cas. Les points ainsi obtenus se superposent rigoureusement, à la précision relative de lecture, à ceux de la compression. Dans le domaine de pressions que nous avons exploré nous n'avons pas observé de phénomène de densification parfois mentionné dans la littérature.

Plus de trente échantillons ont été testés. L'ensemble des résultats conduit aux conclusions suivantes :

a) Les mesures de compressibilité sont indépendantes de la fréquence.

b) Les résultats à la compression et à la décompression sont identiques.

c) La nature du fluide compresseur n'influe pas sur les mesures d'inductance. Il est naturellement indispensable d'employer un liquide isolant. Ainsi des déterminations faites dans les milieux compresseurs suivants : n-pentane, iso-pentane, mélange équivolumétrique de ces derniers ou en recouvrant le bobinage d'une faible couche d'araldite polymérisée, ont donné des résultats ne présentant aucune différence décelable. d) Les courbes $\Delta V / V_{0}=f(P)$ sont toutes linéaires. Seules les pentes varient parfois légèrement d'une expérience à l'autre. Cette différence n'est pas imputable aux mesures des variations d'inductance mais à la détermination expérimentale de $L_{0}$. L'erreur de mesure sur ce terme intervient à la fois au numérateur et au dénominateur de la quantité $L_{0}-L / L_{0}$ qui permet de déduire directement la compressibilité. Il s'agit de la principale cause d'erreur de la méthode.

La figure 4 illustre l'ensemble des résultats obtenus. La courbe continue correspond à la meilleure droite obtenue par la méthode des moindres carrés appliquée à 30 expériences différentes. Les points extérieurs à cette droite correspondent aux mesures les

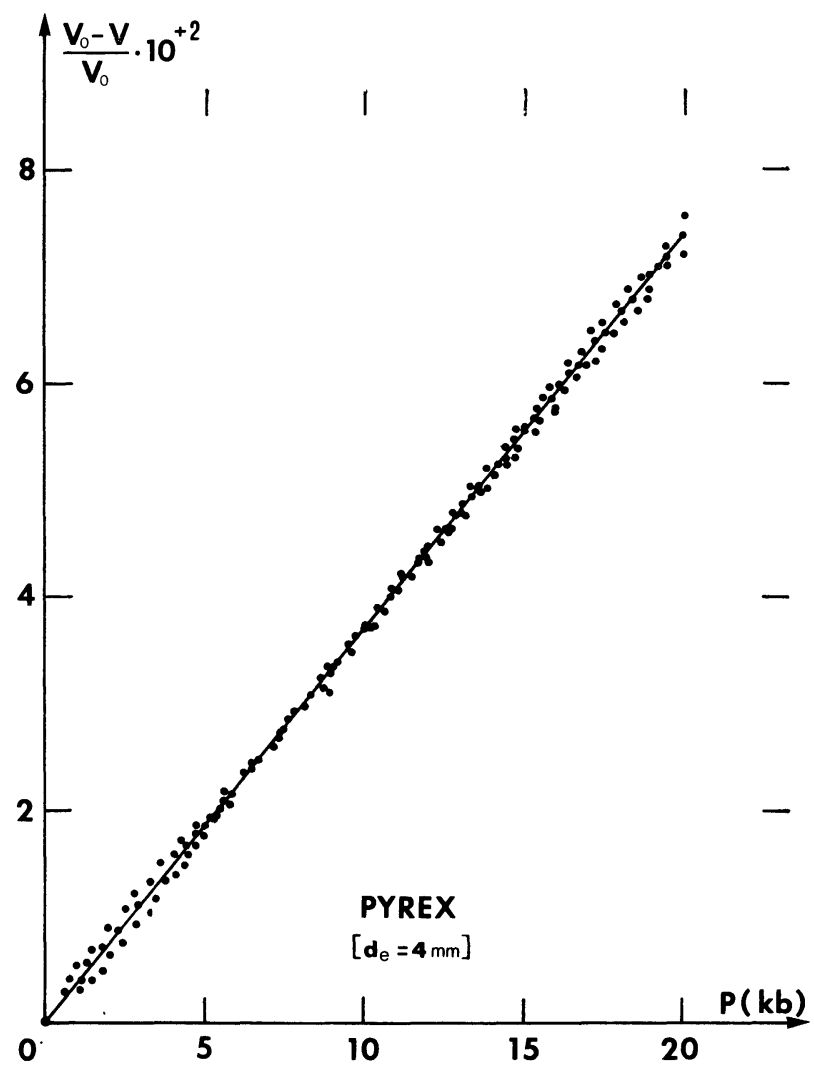

Fig. 4. - Variations de volume du Pyrex en fonction de la pression.

plus aberrantes. La pente de cette droite, qui représente la compressibilité du Pyrex, conduit au résultat suivant :

$$
\Delta V / V_{0} . P=(3,7 \pm 0,1) 10^{-3} / \mathrm{kbar} .
$$

On constate que l'écart à la linéarité, en variation relative de volume, présente une très faible tendance à augmenter avec la pression. Néanmoins il n'excède pas $0,2 \%$ à $20 \mathrm{kbar}$. Par ailleurs l'analyse détaillée des résultats expérimentaux montre que la précision des mesures est meilleure que $3 \%$.

6. Discussion. - La comparaison de nos résultats avec ceux de la littérature est assez difficile du fait 
des différences de composition chimique qui peuvent exister entre les différents types de Pyrex. Seules les tendances et les ordres de grandeurs pourront être confrontées. Cette confrontation est rendue encore plus délicate par le fait que le Pyrex n'a fait l'objet que d'un nombre très limité d'études sous pression. Les premières mesures, dues à Bridgman [27, 28], datent de 1924. En 1931, Adams [29] aborda également ce problème. Enfin, Bridgman [30, 31], en 1948 étendit jusqu'à $100 \mathrm{kbar}$ son premier domaine de mesure.

Ces expérimentateurs notent une légère augmentation de la compressibilité avec la pression. Le comportement redevient normal, selon Bridgman, pour des pressions de l'ordre 28-29 kbar à partir desquelles la compressibilité commence à décroître.

Dans la gamme de pressions commune, 0-12 kbar, ils ont traduit les variations relatives de volume par une équation quadratique de la forme :

$$
\frac{\Delta V}{V_{0}}=a P+b P^{2}
$$

dans laquelle $P$ est exprimé en kbar et $b$ est un coefficient positif.

$$
\begin{aligned}
& \text { Pour Bridgman } \\
& a=3,037 \times 10^{-3} / \mathrm{kbar}, \quad b=8,6 \times 10^{-6} / \mathrm{kbar} .
\end{aligned}
$$

\section{Pour Adams}

$a=3,048 \times 10^{-3} / \mathrm{kbar}, \quad b=7,1 \times 10^{-6} / \mathrm{kbar}$.

Nos résultats

$$
a=3,7 \times 10^{-3} / \mathrm{kbar}, \quad b=0 .
$$

En règle générale, le coefficient quadratique $b$ est nul ou négatif. Le fait qu'il ait été trouvé positif pour le Pyrex est difficilement explicable par des propriétés intrinsèques de ce corps. Ces auteurs ne donnent d'ailleurs aucune explication. Toutefois il faut noter qu'il est très faible de sorte que l'écart à la linéarité l'est aussi (de l'ordre de $1 \%$ entre 0 et $20 \mathrm{kbar}$ ). En revanche, l'écart entre nos mesures et celles de Bridgman est relativement important, de l'ordre de $15 \%$. Il est sensiblement constant - à l'écart de linéarité près - jusqu'à 20 kbar. Cette différence doit être imputée à la nature des matériaux utilisés de part et d'autre. Le Pyrex employé par Bridgman avait été élaboré spécialement pour lui par la Corning
Glass Works alors que nous avons utilisé des baguettes commerciales fabriquées par Sovirel. Les compositions chimiques annoncées par ces fabricants sont assez différentes. Une comparaison absolue ne serait possible qu'avec des corps ayant des compositions chimiques identiques, les échantillons ayant subi des traitements semblables.

7. Conclusions. - Expérimentalement la méthode a été testée jusqu'à des pressions de $20 \mathrm{kbar}$. Les résultats obtenus sur le verre Pyrex nous ont montré qu'elle est extrêmement sensible et reproductible. Une précision de l'ordre de $3 \%$ peut en être attendue. Rappelons que, dans le même domaine de pressions, les diverses autres méthodes ne permettent de mesurer la compressibilité des solides, dans les cas les plus favorables, qu'avec une précision de l'ordre de $5 \%$. Toutefois, une telle amélioration dans la précision des mesures n'est possible que si les conditions suivantes sont respectées :

- Le coefficient de surtension du bobinage doit être aussi élevé que possible. La valeur absolue de la résistance influe sur la sensibilité de la méthode, il est important de la réduire dans toute la mesure du possible.

- Le positionnement des connexions électriques doit être maintenu invariable pendant toute la durée de l'expérience. Des variations de configuration entraîneraient l'apparition de fluctuations d'inductance non évaluables.

- La transmission des efforts doit être parfaitement isotrope.

- La mesure in situ de la pression améliore considérablement la précision de détermination de ce paramètre.

- Il est indispensable de réaliser une liaison intime entre bobinage et échantillon de manière à être sûr que les déformations du premier sont bien identiques à celles du second.

- L'interprétation directe des résultats n'est possible qu'avec des échantillons isolants non magnétiques ; c'est-à-dire lorsque l'effet de peau est négligeable.

- Les dimensions de l'échantillon doivent répondre à certaines conditions pour que les interactions vis-àvis des pièces métalliques environnantes n'interviennent pas.

\section{Bibliographie}

[1] Swenson, C. A., " Equation of state of solids : experimental aspects " Les propriétés physiques des solides sous pression. (Edition CNRS) p. 27, Grenoble 1969.

[2] Barsh, G. R. et Chang, Z. P., "Ultrasonic and static equation of state for cerium » Symp. on the accurate Characterization of high pressure environment NBS Special Publication 326, Gaithersburg 1968.

[3] Kutsar, A. P., "Mesure de la compressibilité des corps solides jusqu'à 25 kbar au moyen de jauges de contrain- tes » C. N. L. L., La Métallurgie noire. Moscou 4 (1966) 171-173.

[4] Maury, R., "Compressibilité des composés intermétalliques de terres rares-métaux de transition » $C$. $R$. Hebd. Séan. Acad. Sci. Paris, 271 (1970) 950-953.

[5] Bridgman, P. W., "Compressibilities of solids" The physics of high pressure (Bell \& Sons, LTD-Londres) 1949.

[6] Bridgman, P. W., "The linear compression of various 
single crystals to $30000 \mathrm{~kg} / \mathrm{cm}^{2} »$. Proc. Am. Acad. Sci., 76 (1948) 89-99.

[7] Bridgman, P. W., " Linear compressions to $30000 \mathrm{~kg} / \mathrm{cm}^{2}$ including relatively incompressible substances ». Proc. Am. Acad. Sci., 77 (1949), 189-234.

[8] Stewart, J. W., In modern high pressure technologie (Edited by R. Wentorf, p. 181, Butterworths, Washington, 1962.

[9] Stewart, J. W., "The compression of solid neon, argon and kripton to 20 kbar». J. Phys. Chem. Solids, 20 (1968) 641-651.

[10] Vaidya, S. N., \& Kennedy, G. C., "Compressibility of 27 metals to $45 \mathrm{kbar} »$ J. Phys. Chem. Solids 31 (1970) 2329-2345.

[11] Vaidya, S. N., \& Kennedy, G. C., "Compressibility of 27 halides to 45 kbar». J. Phys. Chem. Solids, 32 (1971) 951-964.

[12] Perrez-Albuerne, E. A., Forsgren, K. F. \& Drickamer, H. G., "Apparatus for X-ray measurements at very high pressure». Rev. Sci. Instrum. 35 (1964) 29.

[13] LYNCH, R. W. \& DRICKAMER, H. G., « Effect of presure on the lattice parameters of iodine, stannic iodine and p-di-iodobenzene » J. Chem. Phys., 45 (1966) 1020.

[14] Freud, P. J. \& Sclar, C. B., " Apparatus of High Pressure, High Temperature X-ray powder diffraction studies to $100 \mathrm{kbar}$ and $1000{ }^{\circ} \mathrm{C} »$. Abstracts for 1967 , Geol. Soc. Am. Spec., Paper 115 (1968).

[15] Freud, P. J. \& Sclar, C. B., « Apparatus for high pressure high temperature X-ray powder diffraction studies». Rev. Sci. Instrum. 40 (1969) 434-437.

[16] Buchanan, J. Y., "Preliminary note on the compressibility of glass 》 Trans. Roy. Soc. Edin., 29 (1880) 589598.

[17] Bridgman, P. W., "The linear compression of iron to 30000 kg/cm² $/$ Proc. Am. Acad. Arts Sci. 74 (1940) 11-20.

[18] Lincoln, R. C. \& Ruoff, A. L., «Absolute lengh measurement at high pressure » Rev. Sci. Instrum. 44 (1973) 1239-1246.
[19] Grover F. W., «Inductance calculations working formulas and tables » (Dover Publications, Inc., N. Y.) 1946, p. 143 et 164.

[20] Giardini, A. A., Poindexter, E. H. \& Samara, G. A., "High pressure polymorphic transitions studied by audio and radio frequency techniques» Rev. Sci. Instrum. 35 (1964) 713-719.

[21] BarnetT \& Bosco, «Viscosity measurements on liquids to pressure of $60 \mathrm{kbar} » J$. Appl. Phys. 40 (1969) 3144.

[22] Bridgman, P. W., "Physics of high pressure» (Bell \& Sons, LTD, Londres) 1952.

[23] Newhall, D. H., "Manganin high pressure sensors" Instruments and Control Systems, Nov. 1962.

[24] Ivanova, Y. A. \& Tuanova, E. M., " Manganin resistance gages as accurate instruments for high pressure measurements " Symp. on the accurate characterization of high pressure environment. Special Publication NBS 326 Gaithersburg 1968.

[25] Giardini, A. A., Poindexter, E. H., Samara, G. A. \& BARNETT, J., "Compressibility measurement at high pressure by the inductance coil method" Rev. Sci. Instrum. 36 (1965), 1742-1746.

[26] Grover, F. W., Inductance calculations working formulas and tables (Dover Publications N. Y.) 1946, 128.

[27] BRIDGmaN, P. W., " The thermal conductivity and compressibility of several rocks under high pressure " $\mathrm{Am}$. J. Sci., 7 (1924) 81-102.

[28] BRidgman, P. W., «The compressibility of several artificial and natural glasses " Am. J. Sci. 10 (1925) 359-367.

[29] Adams, L. H. \& Gibson, R. E., « The cubic compressibility of certain substances » J. Wash. Acad. Sci., 21 (1931) 381-390.

[30] Bridgman, P. W., "The compression of 39 substances to $100000 \mathrm{~kg} / \mathrm{cm}^{2}$ » Proc. Am. Acad. Arts Sci., 76 (1948) 55-70.

[31] BRIDGman, P. W., " Rough compressions of 177 substances to $40000 \mathrm{~kg} / \mathrm{cm}^{2}$ » Proc. Am. Acad. Arts Sci. 76 (1948) 71-87. 